\title{
MESON RESONANCES IN PROTON-ANTIPROTON ANNIHILATION
}

\author{
C. AMSLER \\ Physik-Institut der Universität Zürich \\ Winterthurerstrasse 190, CH-8035 Zürich, Switzerland \\ E-mail:Claude.Amsler@cern.ch
}

\begin{abstract}
Crystal Barrel data for proton-antiproton annihilation in flight at $900 \mathrm{MeV} / \mathrm{c}$ are presented. The channels $p \bar{p} \rightarrow 3 \pi^{0}, \pi^{0} \pi^{0} \eta$ and $\pi^{0} \eta \eta$ are used to search for isoscalar $0^{++}$and $2^{++}$mesons in the mass range $1500-2000 \mathrm{MeV}$. Both $3 \pi^{0}$ and $\pi^{0} \eta \eta$ data sets require an isoscalar $2^{++}$resonance decaying into $\pi^{0} \pi^{0}$ and $\eta \eta$ with mass $M=(1867 \pm 46) \mathrm{MeV}$ and width $\Gamma=(385 \pm 58) \mathrm{MeV}$. The analysis of $\pi^{0} \pi^{0} \eta$ leads to an isovector $2^{++}$state decaying into $\pi^{0} \eta$ with mass $M=(1698 \pm 44) \mathrm{MeV}$ and width $\Gamma=(265 \pm 55) \mathrm{MeV}$. The $3 \pi^{0}$ and $\pi^{0} \eta \eta$ data do not show any $f_{0}(1710)$. This adds supportive evidence that this meson is mainly $s \bar{s}$.
\end{abstract}

\section{Introduction}

Scalar $\left(0^{++}\right)$mesons overpopulate the mass spectrum below $2 \mathrm{GeV}$. Table 1 shows a possible $\mathrm{SU}(3)$ classification of these states. The low mass scalars are interpreted as scattering resonances ${ }^{1}$. Alternatively, the $a_{0}(980)$ and $f_{0}(980)$ are often referred to as $K \bar{K}$ molecules or $q^{2} \bar{q}^{2}$ states $^{2,3}$. In the literature the narrow $f_{0}(1500)$ and $f_{0}(1710)$ compete for being the ground state glueball. Recent data in $\bar{p} p$ annihilation and in central production show that both the $f_{0}(1370)$ and $f_{0}(1500)$ couple mainly to pions ${ }^{4,5}$ while $f_{0}(1710)$ couples mainly to kaons ${ }^{5}$. I will show that $f_{0}(1710)$ is dominantly $s \bar{s}$. This then adds evidence for $f_{0}(1500)$ to be mainly gluonic, while $f_{0}(1370)$ is the $u \bar{u}+d \bar{d}$ isoscalar meson 6 . More complicated schemes have been proposed. For a detailed discussion and for a bibliography see refs. ${ }^{7,8}$.

\begin{tabular}{|c|c|c|c|c|}
\hline$I=1$ & $I=0$ & $I=0$ & $I=1 / 2$ & Nature \\
\hline$a_{0}(980)$ & $\begin{array}{c}f_{0}(400-1200) \\
(\text { or } \sigma)\end{array}$ & $f_{0}(980)$ & $\kappa(900)$ & Scattering resonances \\
\hline$a_{0}(1450)$ & $\begin{array}{c}f_{0}(1370) \\
f_{0}(1500)\end{array}$ & $f_{0}(1710)$ & $K_{0}^{*}(1430)$ & $1^{3} P_{0}(q \bar{q})$ \\
\hline$?$ & $f_{0}(2020)$ & $f_{0}(2200)$ & $K_{0}^{*}(1950)$ & $2^{3} P_{0}(q \bar{q})$ \\
\hline
\end{tabular}

Table 1: Tentative SU(3) assignment of scalar mesons. The first isoscalar (second column) couples strongly to pions, the second (third column) strongly to kaons. The "pionic" states are very broad, except $f_{0}(1500)$, which appears to be supernumerary. 
The tensor $\left(2^{++}\right)$ground state nonet is well established, but a tentative classification of $2^{++} q \bar{q}$ excitations, which lie above $1500 \mathrm{MeV}$, is not possible. This is due to the lack of systematic data in two-body decays like $\pi \pi, K \bar{K}$ and $\eta \eta$, in contrast to the low mass region below $1500 \mathrm{MeV}$, which was extensively studied at LEAR. The $2^{++}$glueball is predicted in the $2200 \mathrm{MeV}$ region ${ }^{9}$.

\section{Annihilation at $900 \mathrm{MeV} / \mathrm{c}$}

This paper presents the analyses of proton-antiproton annihilation data at 900 $\mathrm{MeV} / \mathrm{c}$, corresponding to a c.m.s. energy of $2050 \mathrm{MeV}$, into the three final states $\pi^{0} \pi^{0} \pi^{0}, \pi^{0} \pi^{0} \eta$ and $\pi^{0} \eta \eta$, leading to six detected photons ${ }^{10}$.

The analysis is based on 17.9 million events which were taken with the all-neutral trigger during the last data taking run of Crystal Barrel at LEAR in 1996. The offline analysis required completely reconstructed events with exactly six clusters and no charged tracks.

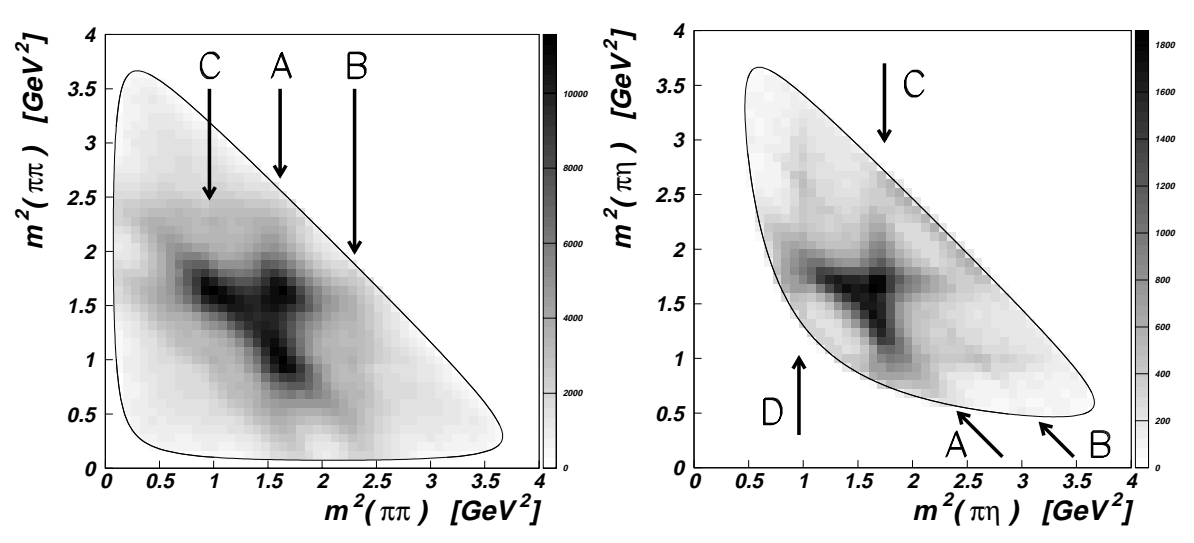

Figure 1: Left: Dalitz plot for $p \bar{p} \rightarrow 3 \pi^{0}$ (six entries per event) with $f_{2}(1270)(\mathrm{A}), f_{0}(1500)$ (B), $f_{0}(980)$ (C). Right: Dalitz plot for $p \bar{p} \rightarrow \pi^{0} \pi^{0} \eta$ (two entries per event) with $f_{2}(1270)$ $(\mathrm{A}), f_{0}(980)(\mathrm{B}), a_{2}(1320)(\mathrm{C})$ and $a_{0}(980)(\mathrm{D})$.

The three annihilation channels were selected by applying kinematic fits requiring also three pairs of $2 \gamma$ invariant masses to match the $\pi^{0}$ or $\eta$ masses. For measurements in flight the annihilation vertex of neutral events was not observed and had therefore to be determined by the kinematic fit. The data and results presented here are at variance with the ones reported in ref. ${ }^{11}$ which assumed a vertex at the center of the detector.

The feedthrough from one channel to the other was determined to be less than $0.7 \%$. The reaction $\bar{p} p \rightarrow \pi^{0} \omega$ with $\omega \rightarrow \pi^{0} \gamma$ (and a missing $\gamma$ ) 

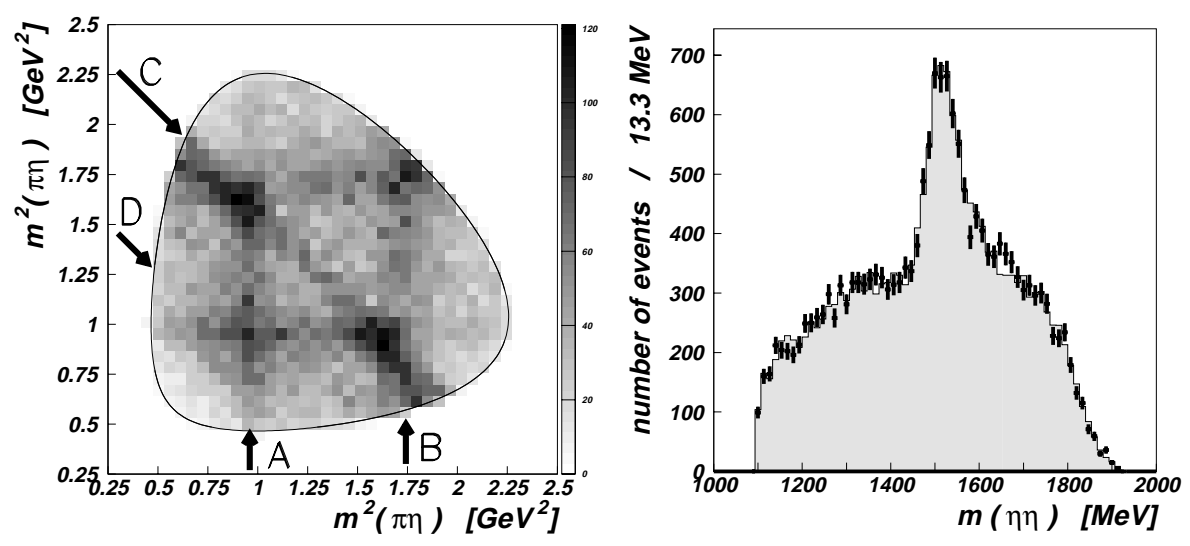

Figure 2: Left: Dalitz plot for $p \bar{p} \rightarrow \pi^{0} \eta \eta$ (two entries per event) with $a_{0}(980)$ (A), $a_{2}(1320)$ (B), $f_{0}(1500) / f_{2}^{\prime}(1525)(\mathrm{C})$. Right: $\eta \eta$ mass projection showing the $f_{0}(1500) / f_{2}^{\prime}(1525)$. The long tail is due to the new $f_{2}(1870)$. The histogram is the final fit discussed in the text.

was the dominating background channel for $\pi^{0} \eta \eta$ and $\pi^{0} \pi^{0} \eta$. Background contributions of $13 \%$ to $\pi^{0} \eta \eta$ and $3 \%$ to $\pi^{0} \pi^{0} \eta$ were estimated from the data. The background in the $3 \pi^{0}$ channel was negligible.

The symmetrised $\pi^{0} \pi^{0} \pi^{0}$ Dalitz plot is shown in fig. 1 (left). One observes the $f_{2}(1270)$, the $f_{0}(1500)$ and/or $f_{2}(1565)$. A faint dip in the $1500 \mathrm{MeV}$ band around $1000 \mathrm{MeV}$ corresponds to the $f_{0}(980)$ interfering destructively with the structure at $1500 \mathrm{MeV}$. Figure 1 (right) shows the Dalitz plot for $\pi^{0} \pi^{0} \eta$. One observes the $f_{2}(1270)$, the $f_{0}(980)$, the $f_{2}(1270)$, and the $a_{0}(980)$. The symmetrised $\pi^{0} \eta \eta$ Dalitz plot and the $\eta \eta$ mass projections are shown in Figure 2. One observes the $a_{0}(980)$, the $a_{2}(1320)$, the $f_{0}(1500)$, and/or $f_{2}^{\prime}(1525)$, and a band around $1000 \mathrm{MeV}$ from the $a_{0}(980)$. In the region of the $f_{0}(1710)$, only the interference of the two $a_{0}(980)$ bands can be observed and no obvious signal is present (arrow D).

The data sets were analysed in the helicity formalism in terms of the isobar model, in which the $\bar{p} p$ system is assumed to decay into the three-body final states through a two-body intermediate state made of a resonance and a spectator meson ${ }^{12}$. The K-matrix formalism described the mass dependance of resonances ${ }^{13}$. Masses and widths of resonances were given by the complex poles of the $T$-matrix.

The following initial partial waves were included in the analysis of the present data: ${ }^{1} S_{0},{ }^{3} P_{1},{ }^{3} P_{2}+{ }^{3} F_{2},{ }^{1} D_{2}$, and ${ }^{3} F_{3}$. The $\pi \pi \pi \eta$ and $\eta \eta$ S-waves were parametrized by $\mathrm{K}$-matrices with the parameters obtained at rest, where 
only ${ }^{1} S_{0}$ contributes ${ }^{14,15}$.

\section{Results}

The first description of $3 \pi^{0}$ included the $\pi \pi$ S-wave and the $f_{2}(1270)$. The fit was then extended with a second pole in the $\pi \pi$ D-wave to test for a spin 2 meson. The fit clearly required a high-mass tensor state around $1870 \mathrm{MeV}$. The best fit was obtained by parametrising the $\pi \pi$ D-wave as a K-matrix with three poles: $f_{2}(1270), f_{2}(1565)$ and a broad new tensor state with mass 1877 $\mathrm{MeV}$ and width $318 \mathrm{MeV}$, which we call $f_{2}(1870)$.

The first description of $\pi^{0} \pi^{0} \eta$ consisted of the $\pi \pi$ S-wave, the $f_{2}(1270)$, the $a_{0}(980)$ and $a_{0}(1450)$ in the $\pi \eta \mathrm{S}$-wave, and the $a_{2}(1320)$. Crystal Barrel reported in $\pi^{0} \eta \eta$ at $1940 \mathrm{MeV} / \mathrm{c}$ an isovector state at $1660 \mathrm{MeV}$, decaying into $\pi^{0} \eta^{16}$. Hence this $a_{2}(1660)$ was introduced into the $(1 \times 1) \mathrm{K}$-matrix of the $\pi \eta \mathrm{D}$-wave as a second pole. The fit improved significantly. The $a_{2}(1660)$ contributes with $(7 \pm 2) \%$ to the data and for this $2^{++}$isovector state we find a mass of $1698 \pm 44 \mathrm{MeV}$ and a width of $265 \pm 55 \mathrm{MeV}$, in excellent agreement with our result in $\pi^{0} \eta \eta$ at $1940 \mathrm{MeV} / \mathrm{c}^{16}$.

The first description of $\pi^{0} \eta \eta$ included in the $\pi \eta$ S-wave, the $a_{0}(980)$ and the $a_{0}(1450)$, and the $a_{2}(1320)$ in the $\pi \eta \mathrm{D}$-wave. Significant differences between fit and data were observed for $\eta \eta$ masses around $1500-1550 \mathrm{MeV}$ and $1650-1800$ $\mathrm{MeV}$. To describe the $\eta \eta$ peak at $1500 \mathrm{MeV}$, the tensor resonance $f_{2}^{\prime}(1525)$ was introduced. A good agreement with data could be obtained in the high mass region by adding a high mass tensor state with mass $1820 \mathrm{MeV}$ and width $358 \mathrm{MeV}$. The mass and width of the latter agree with the ones found in the analysis of $\pi^{0} \pi^{0} \pi^{0}$.

The $\eta \eta$ invariant mass projection is shown in fig. 2. The data description is good and there are no significant deviations. The $f_{0}(1500)$ contributes with $(10 \pm 2) \%$, the $f_{2}^{\prime}(1525)$ with $\left(15_{-3}^{+1}\right) \%$. The inclusion of the $f_{0}(1710)$ in the $\eta \eta \mathrm{S}$-wave was not successful. Hence the $f_{0}(1710)$ is not required to describe the $\pi^{0} \eta \eta$ data set.

Since the analyses of $\pi^{0} \pi^{0} \pi^{0}$ and $\pi^{0} \eta \eta$ require a high-mass isoscalar tensor state, the two data sets were simultaneously fitted with a common description of the resonances, e.g. the $f_{2}(1870)$ (for $\pi^{0} \pi^{0} \eta$ this state lies far above the phase space limit).

The fitted Dalitz plots and projections for the two data sets differ only marginally from the ones obtained by the single fits. The T-matrix mass and width of the isoscalar $f_{2}(1870)$ are $1867 \pm 46 \mathrm{MeV}$ and $385 \pm 58 \mathrm{MeV}$, respectively. 


\section{Discussion and conclusions}

The analysis of $\bar{p} p \rightarrow \pi^{0} \pi^{0} \eta$ clearly showed that the $\pi \eta$ D-wave wave requires two poles, corresponding to the $a_{2}(1320)$ and its radial excitation $a_{2}(1660)$. The L3 collaboration analysing $\gamma \gamma \rightarrow \pi^{+} \pi^{-} \pi^{0}$ also reported a $2^{++}$isovector state at a mass of $1750 \mathrm{MeV}$, decaying into $\pi^{+} \pi^{-} \pi^{0} 17$. The mass of our $a_{2}(1660)$ is consistent with the L3 result within errors.

The fits of $\pi^{0} \pi^{0} \pi^{0}$ and $\pi^{0} \eta \eta$ including an $f_{0}(1710)$ were not satisfactory. In the best fit of $\bar{p} p \rightarrow 3 \pi^{0}$ the improvement of the log-likelihood was not significant when the $f_{0}(1710)$ was included. When mass and width of the $f_{0}(1710)$ were fitted freely in the $\pi^{0} \pi^{0} \pi^{0}$ and $\pi^{0} \eta \eta$ data sets, the resulting object was broad and the other resonances became unstable. The conclusion was drawn that the $f_{0}(1710)$ is not present. We derived an upper limit for the contribution of the $f_{0}(1710)$ to $\pi^{0} \pi^{0} \pi^{0}$ and $\pi^{0} \eta \eta$ of $2.1 \%$ and $2.6 \%$, respectively, at $90 \%$ confidence level, assuming a mass of $1715 \mathrm{MeV}$ and a width of $125 \mathrm{MeV}^{18}$.

The absence of a signal for this isoscalar points to a dominant $s \bar{s}$ component, as there are no known mechanisms suppressing $u \bar{u}+d \bar{d}$ scalars in $\bar{p} p$ annihilation. The absence of $f_{0}(1710)$ in our data is therefore compatible with an $s \bar{s}$ assignment. Recent results in central production ${ }^{5}$ show that $f_{0}(1710)$ prefers to decay into $K \bar{K}$ rather than into $\pi \pi$. This also points to an $s \bar{s}$ interpretation of the $f_{0}(1710)$ and therefore suggests that this meson is the (mainly) $s \bar{s}$ member of the scalar nonet.

New data in $\gamma \gamma$ collisions have been presented by the LEP collaborations. L3 observes three peaks below $2 \mathrm{GeV}$ in the $K_{S} K_{S}$ mass distribution ${ }^{19,20}$. The lowest peak corresponds to $f_{2}(1270)$ and $a_{2}(1320)$, interfering destructively. The amplitude analysis reveals the $f_{2}^{\prime}(1525)$ while $f_{0}(1500)$ is not observed. Spin 2 is preferred for the third peak around $1760 \mathrm{MeV}$. However, a large spin 0 component (as large as 50\%) can be accommodated ${ }^{20}$. Note that the isospin is not determined in the $K_{S} K_{S}$ final state. We suggest that the $2^{++}$ state observed in $\gamma \gamma \rightarrow K_{S} K_{S}$ is actually $a_{2}(1660)$ and that the smaller $0^{++}$ contribution is due to $f_{0}(1710)$.

These results in $\bar{p} p, \gamma \gamma$ and central collisions strengthen the interpretation of the $f_{0}(1500)$ as a glueball, or as a state with a large gluonic admixture in its wave-function ${ }^{6,21}$ : since $f_{0}(1500)$ does not couple strongly to $K \bar{K}$ it absence in $\gamma \gamma \rightarrow K_{S} K_{S}$ is not surprising. However, the ALEPH collaboration, studying the reaction $\gamma \gamma \rightarrow \pi^{+} \pi^{-}$, observes $f_{2}(1270)$ but not $f_{0}(1500)^{22}$. Since $f_{0}(1500)$ has a large coupling to $\pi \pi$ this, together with its absence in $K_{S} K_{S}$, indicates that $f_{0}(1500)$ is not produced in $\gamma \gamma$ processes, as expected if $f_{0}(1500)$ is mostly gluonic. We note that ALEPH does not observe $f_{0}(1710)$ either, but the data 
are not sensitive enough for $s \bar{s}$ states.

The $\pi^{0} \pi^{0} \pi^{0}$ and $\pi^{0} \eta \eta$ data sets require a high-mass tensor $f_{2}(1870)$ decaying to $\pi^{0} \pi^{0}$ or $\eta \eta$. We do not confirm the narrow $f_{2}(1810)$ decaying into $\pi \pi^{18}$. What is this new state? The relative strength of the $f_{2}(1870)$ decaying to $\eta \eta$ and $\pi^{0} \pi^{0}$ is $0.27 \pm 0.10$. This ratio is related to $\mathrm{SU}(3)$ mixing angles 6 . One gets two solutions for the mixing angle ${ }^{10}$, one being compatible with a pure $u \bar{u}+d \bar{d}$ state $\left(\theta \sim 35.3^{\circ}\right)$, hence a radial excitation of the $f_{2}(1270)$. Then $f_{2}(1565)$ and $f_{2}(1870)$ are both radial excitations but do not belong to the same nonet. The other solution $\left(\theta \sim 15^{\circ}\right)$ leads to a large $s \bar{s}$ component for $f_{2}(1870)$, in which case $f_{2}(1565), a_{2}(1660)$ and $f_{2}(1870)$ could belong to the $2^{3} P_{2}$ nonet of radial excitations. The mass formula then predicts the kaon-like state to lie around $1800 \mathrm{MeV}$. The ambiguity between the two mixing angles could be resolved by measuring the $\eta \eta$ and/or $K \bar{K}$ decay rates of the $f_{2}(1870)$.

1. J.A. Oller et al., Phys. Rev. D 59 (1999) 074001

2. R.L. Jaffe, Phys. Rev. D 15 (1977) 267, 281

3. J. Weinstein, N. Isgur, Phys. Rev. D 41 (1990) 2236

4. A. Abele et al., Phys. Rev. D 57 (1998) 3860

5. D. Barberis et al., Phys. Lett. B 462 (1999) 462

6. C. Amsler and F. E. Close, Phys. Rev. D 53 (1996) 295

7. C. Amsler, Nucl. Phys. A 663 (2000) 93c

8. C. Amsler in Rev. of Particle Physics, Eur. Phys. J. C 15 (2000) 682

9. C. Michael, Hadron 97 Conf., AIP Conf. Proc. 432 (1998) 657

10. C. Amsler et al., submitted to Eur. Phys. J. C; M. Heinzelmann, PhD thesis, Universität Zürich, 2000

11. A. V. Anisovich et al., Phys. Lett. B 449 (1999) 145, 154

12. C. Amsler and J.C. Bizot, Comp. Phys. Commun. 30 (1983) 21

13. S. U. Chung et al., Ann. Physik (Leipzig) 4 (1995) 404

14. C. Amsler et al., Phys. Lett. B 355 (1995) 425

15. For a review see C. Amsler, Rev. Mod. Phys. 70 (1998) 1293

16. A. Abele et al., Eur. Phys. J. C 8 (1999) 67

17. M. Acciarri et al., Phys. Lett. B 413 (1997) 147

18. D. E. Groom et al., Rev. of Part. Physics, Eur. Phys. J. C 15 (2000) 1

19. M. Acciarri et al., Phys. Lett. B 501 (2001) 173

20. S. Braccini, PhD thesis, Université de Genève, 2001

21. F. Close and A. Kirk, Phys. Lett. B 483 (2000) 345

22. R. Barate et al., Phys. Lett. B 472 (2000) 189 